\title{
Knowledge on insulin therapy among healthcare professionals
}

\author{
An evaluation based on pre and post test analysis of a training programme on \\ insulin therapy \\ C L Weeraratne ${ }^{1}$, W K de Abrew ${ }^{1}$, R P Kuruppumullage ${ }^{1}$ \\ Journal of the Ceylon College of Physicians, 2010, 41, 67-71
}

\section{General objective}

To identify deficient areas in the knowledge on insulin therapy among healthcare professionals who manage patients on insulin and assess the improvement in their knowledge following a training programme.

\section{Background}

Daily administration of subcutaneous insulin is essential in the management of type 1 diabetes. In type 2 diabetes subcutaneous insulin is required under certain situations such as acute severe infections, acute myocardial infarction, during peri-operative management and in oral antidiabetic treatment failure. Also, insulin is required by many diabetic women during pregnancy.

Healthcare professionals need to pay attention to details of subcutaneous insulin therapy in order to improve patient compliance to therapy and achieve optimum blood glucose control. In this regard knowledge and skills regarding selecting suitable injecting devices, the correct injection technique, and the correct storage of insulin is essential. Continuous education of healthcare professionals is required to ensure adequacy of knowledge, skills and attitudes for quality care of people with diabetes.

\section{Design and setting}

A training module on insulin therapy was developed as a component of Health Sector Development project of Ministry of Healthcare and Nutrition.

The first training programme of this module was conducted in the month of July 2009 for a group of healthcare professionals in one province of Sri Lanka. This was intended as a training of trainers session. There were 51 doctors of different grades from junior doctors to consultants and 43 nurses of different grades as participants.

The training programme included a PowerPoint presentation with photographs and video clips on different insulin preparations, injecting devices and steps of subcutaneous insulin injection. The programme also included a video presentation of a diabetic patient on insulin. The objectives of the video were to highlight the importance of educating patients and their care givers on different aspects of insulin therapy, to reinforce technical aspects of subcutaneous insulin administration and to highlight the importance of multidisciplinary care of such patients.

\section{Results}

Of the 94 participants $52(55.3 \%)$ completed the pretest and 51(54.3\%) completed the post test.

The results are given in Table 1. The correct answer to each of the statement is also indicated as $T / F$. In addition, $p$ values were calculated to see if the improvement in the knowledge following the training session is statistically significant at $p<0.05$.

\footnotetext{
${ }^{1}$ Department of Pharmacology, Faculty of Medicine, University of Colombo.
} 
Table 1. Percentages of correct responses on topics related to insulin therapy

The questions and the corresponding responses given in the questionnaire. The true and false responses are indicated as $(T)$ and $(F)$

Percentage knowing the correct answer

Pre-test Post-test P value

\section{Knowledge regarding insulin}
A Insulin is secreted by the alpha cells of the pancreas (F)
$65.4 \%$
$74.5 \%$
0.3
$B$ The insulin molecule consists of 2 chains of amino acids ( $T$ )
$50 \%$
$80.4 \%$
0.001
C Insulin is secreted mainly as proinsulin (F)
$13 \%$
$33.3 \%$
0.03
D Insulin is a catabolic hormone (F)
$50 \%$
$56.9 \%$
0.49
E Insulin reduces entry of glucose into muscle $(F)$
$57.7 \%$
$56.9 \%$
0.93

\section{Knowledge regarding soluble insulin}
A Soluble Insulin is an opaque solution (F)
$50 \%$
$60.8 \%$
0.27
B Soluble Insulin can be given intravenously ( $T$ )
$82.7 \%$
$92.2 \%$
0.15
C Soluble Insulin has a duration of action of 18 hours (F)
$78.9 \%$
$86.3 \%$
0.32
D Soluble insulin is also known as neutral insulin ( $T$ )
$32.7 \%$
$33.3 \%$
0.94
E Soluble Insulin is contraindicated in pregnancy (F)
$67.3 \%$
$80.4 \%$
0.13

\section{Knowledge regarding opaque insulin suspensions -}

The following are opaque insulin suspensions
A Soluble insulin (F)
$48.1 \%$
$58.8 \%$
0.27
$B$ Isophane insulin ( $T$ )
$36.5 \%$
$76.5 \%$
$<0.0001$
C Lente insulin ( $T$ )
$42.31 \%$
$74.5 \%$
0.0009
D Pre mixed biphasic insulin $(T)$
$32.7 \%$
$68.6 \%$
0.0003
E Insulin glargine $(F)$
$23.1 \%$
$33.3 \%$
0.24

\section{Knowledge on insulin administration}
A Insulin is measured in units $(T)$
$88.5 \%$
$84.3 \%$
0.54
B The capacity of an Insulin vial is usually $1 \mathrm{ml}(\mathrm{F})$
$82.7 \%$
$90.2 \%$
0.27
C The recommended gauge of the Insulin needle is 25 gauge $(F)$
$25 \%$
$78.4 \%$
$<0.0001$
D A vial of soluble insulin has 1000 units of insulin (T)
$69.2 \%$
$88.2 \%$
0.018
$E$ The insulin pen has a needle of 31 gauge $(T)$
$40.4 \%$
$88.2 \%$
$<0.0001$

\section{Knowledge on insulin storage}
A Unopened insulin vials should not be refrigerated (F)
$82.7 \%$
$72.6 \%$
0.22
B Unopened insulin vials should not be stored in the freezer compartment of a fridge $(T)$
$75 \%$
$66.7 \%$
0.35 

The insulin vialshould be kept in the door of the fidae (E))
Percentage knowing the correct answer
Pre-test Post-test P value
C The insulin vial should be kept in the door of the fridge (F)
$44.2 \%$
$90.2 \%$
$<0.0001$
$D$ If a fridge is not available it could be stored in a clay pot
$75 \%$
$96.1 \%$
0.002 containing water $(T)$ E The insulin vial in use could be kept at a room temperature
below 25 degrees centigrade $(T)$
$44.2 \%$
$80.4 \%$
0.0002
06 Knowledge regarding site of fastest absorption during subcutaneous administration of insulin
A Left upper arm (F)
$25 \%$
$31.4 \%$
0.47
B Buttock (F)
$32.7 \%$
$51 \%$
0.06
C Thigh $(F)$
$21.1 \%$
$29.4 \%$
0.33
D Abdomen (T)
$61.5 \%$
$82.4 \%$
0.019
E Right upper arm (F)
$21.2 \%$
$39.2 \%$
0.045

07 The knowledge on ways of minimizing pain associated with insulin injections
A Injecting insulin while it is cold (F)
$38.5 \%$
$70.6 \%$
0.001
$B$ Inserting the needle slowly into skin (F)
$21.2 \%$
$70.6 \%$
$<0.0001$
C Removing the needle slowly from the skin (F)
$26.9 \%$
$70.6 \%$
$<0.0001$
D Using a 29 gauge needle $(T)$
$42.3 \%$
$86.3 \%$
$<0.0001$
E Removing air bubbles from the insulin syringe before injecting
(T) $73.1 \%$
$86.3 \%$
0.096

08 The knowledge on symptoms of hypoglycaemia
A Feeling faint $(T)$
$90.4 \%$
$88.2 \%$
0.72
B Headache $(T)$
$67.3 \%$
$80.4 \%$
0.13
C Hunger $(T)$
$65.4 \%$
$72.6 \%$
0.43
D Sweating $(T)$
$90.4 \%$
$90.2 \%$
0.97
E Diarrhoea (F)
$51.9 \%$
$51 \%$
0.92

09 The knowledge on types of rapid acting insulin analogues
A Insulin lispro $(T)$
$19.2 \%$
$58.8 \%$
$<0.0001$
$B$ Insulin glargine $(T)$
$17.3 \%$
$37.2 \%$
0.023
C Soluble insulin (F)
$17.3 \%$
$27.4 \%$
0.22
D Insulin aspart (T)
$13.5 \%$
$64.7 \%$
$<0.0001$
E NPH insulin (T)
$21.2 \%$
$43.1 \%$
0.02

10 The knowledge regarding the sites that need to be wiped with a surgical spirit swab
A The skin of site of the injection $(T)$
$48.1 \%$
$72.6 \%$
0.01 


\begin{tabular}{|c|c|c|}
\hline \multicolumn{3}{|c|}{ Percentage knowing the correct answer } \\
\hline Pre-test & Post-test & $P$ value \\
\hline $38.5 \%$ & $90.2 \%$ & $<0.0001$ \\
\hline $51.9 \%$ & $72.6 \%$ & 0.03 \\
\hline $44.2 \%$ & $68.6 \%$ & 0.012 \\
\hline $25 \%$ & $56.9 \%$ & 0.001 \\
\hline
\end{tabular}

\section{The knowledge regarding preparation for insulin injection}
A The hands should be washed with soap and water before handling injection devices $(T)$
B The insulin vial should be kept at room temperature atleast for 15 minutes before giving injection ( $\mathrm{T}$ )

$17.3 \% \quad 78.4 \% \quad<0.0001$
C Air should be injected into the insulin vial before drawing insulin out of the vial $(T)$
D If drawing both soluble and isophane insulin into the same syringe the isophane insulin should be drawn into the vial first $(F)$
$62.8 \%$
E The site of injection is changed to prevent lypohypertrophy (T)

\section{The knowledge regarding glucometers}
A Glucostrips are not specific to glucometers (F)
$B$ The glucometer need to be calibrated only once during its life time $(F)$
C The glucometer should be standardized using a test solution $(T)$
D The glucometer readings are given only in $\mathrm{mg} / \mathrm{dl}(\mathrm{F})$
E The glucometer strips can be reused $(T)$

13 The knowledge regarding the most appropriate initial management of hypoglycaemia - the following case scenario was given

A sixteen year old boy who is on Insulin therapy for Type I diabetes is admitted to hospital because he suddenly became unconscious. His RBS was found to be $40 \mathrm{mg} / \mathrm{dl}$ on admission
A Oral glucose $(F)$
$36.5 \%$
$43.1 \%$
0.49
B A cup of tea with sugar $(F)$
$36.5 \%$
$43.1 \%$
0.49
C Glucagon intramuscularly $(F)$
$25 \%$
$9.8 \%$
0.04
D A $5 \%$ dextrose drip (F)
$21.2 \%$
$27.4 \%$
0.33
E 50\% dextrose intravenously (T)
$69.2 \%$
$90.2 \%$
0.008

\section{The knowledge on capillary blood glucose measurement}

A Blood glucose strips should match the type of glucometer used $(T)$ 
Percentage knowing the correct answer

B Each time a new supply of blood glucose strips are to be used the machine should be calibrated $(T)$

Pre-test

Post-test

$P$ value

Blood glucose value is displayed in the glucometer, 5 minutes after testing $(F)$

Glucometers are standardized by checking with standard solutions $(\mathrm{T})$

$53.9 \%$

$74.5 \%$

0.03

E Usually a capillary blood sample is used to test in a glucometer ( $\mathrm{T}$ )

\section{Limitations}

The number of respondents was much less than the number of total participants and the study sample would have been more representative had the number been greater. Also, the session as well as pre and post tests were conducted in English which may have affected the comprehension of some participants. Furthermore, we were unable to assess the knowledge of the different categories of health professionals separately with this study and this analysis reflects only the knowledge level of the health professionals in one province of the country. Further more we were able to only analysis the immediate effects of the training programme. We were not able to assess the long term impact of the training program on practical skills development related to insulin injection.

\section{Discussion and Conclusion}

Despite the above mentioned limitations the results of the pre and post test analyses clearly showed an improvement in the level of knowledge on each fact regarding insulin therapy. However, the level of knowledge and its improvement varied between different facts. Consequently, the study indicates the areas that need to be addressed with greater emphasis. The pretest analysis disclosed that the knowledge of the participants on measures to minimize pain associated with insulin injections was especially deficient. However, pain associated with insulin injections is of great relevance since this is a major factor that leads to poor patient compliance to insulin therapy. It further affects the quality of life of patients as well. The post test analysis shows that the training program has been successful in significantly improving knowledge in this regard. The pretest analysis has also shown that the participants knowledge on more recent developments relating to insulin therapy such as the insulin pen device, glucometers and insulin analogues were also limited. Therefore in general the pretest has provided some insight regarding the present knowledge of health professionals regarding insulin therapy and insulin injection technique and has shed light on deficient areas that need to be highlighted and reinforced. This insight would be helpful in planning future educational and training programmes for the rest of the country. The post test results indicate that a training program of this nature is successful in bridging important gaps in the knowledge relating to insulin therapy and insulin injection technique cost effectively within a short period of time.

\section{Acknowledgement}

We acknowledge the contribution made by the Ceylon College of Physicians as well as the Health Sector Development Project in organizing and coordinating this training programme. Also we are thankful to all the participants who engaged in this study.

\section{References}

1. Brown DC, Diabetes mellitus, insulin, oral antidiabetic agents, obesity.In: Bennette PN, Brown MJ. Clinical Pharmacology. 10th edn. Spain: Churchill Livingstone, 2008: 608-26

2. Sweetman $S(E d)$, Martindale: The complete drug reference. London: Pharmaceutical Press. Electronic version (2007).

3. American Diabetic Association. Position statement insulin administration. Diabetes Care 2002; 25: S112-S115.

4. British Medical Association and the Royal Pharmaceutical Society of Great Britain. British National Formulary. London: BMA, RPSGB; 2008: 55. 